\title{
Modelling of Hexagonal Electromagnetic Sensor
}

\author{
Sheetal Vilas Mapare \\ Sant Gadge Baba Amravati \\ University, Amravati, Vidyalankar Institute of \\ Technology,Wadala, Mumbai
}

\author{
G. G. Sarate, PhD \\ Sant Gadge Baba Amravati \\ University, Amravati, Government Polytechnic, \\ Yavatmal
}

\begin{abstract}
This paper introduces hexagonal shape sensors that is a meander and mesh type. Modelling, simulation and development of Hexagonal Sensor and the results are discussed in this paper. The characteristics of hexagonal spiral inductor and interdigital capacitor are determined. The hexagonal spiral inductor is a new electronic sensor that can be used for various measurements that produces magnetic field. The interdigital capacitor has been designed with two different widths of electrode results in electric field generation. This hexagonal spiral inductor and interdigital sensor are combined to form one single hexagonal electromagnetic sensor, interdigital sensor at the centre of the spiral. The developed structure is double sided with hexagonal spiral inductor and interdigital sensor on top and ground on the other side. The sensor is designed using COMSOL Multiphysics software to calculate sensors' characteristics and it has been fabricated on FR4 substrate of thickness $0.8 \mathrm{~mm}$.
\end{abstract}

\section{Keywords}

Sensor, Electromagnetic, Electronic Sensor, Hexagonal spiral Inductor, Impedance.

\section{INTRODUCTION}

Electromagnetic sensors are novel yet rapidly developing technology. The sensors have been designed, fabricated and employment successfully. These are successfully used in applications such as the printed circuit boards assessment $[1,2,3]$, near surface material properties estimation [4,5], electroplated materials [6,7], saxophone reed examination[8], dairy products inspection[7]. The planar interdigital sensors have been used for the non-invasive monitoring of fat content and bodily fluids [9].

The sensor either may be of a meander or of a mesh type or combination of both, the sensors selection depends on the applications. The use of Meander type sensing coils for testing the integrity of materials has been reported in [10]. If the meander type sensor is used to detect cracks in a metal, the disadvantage is that the performance of the sensor is dependent of the alignment of the cracks or non-homogeneity of the material structure (in the progression of evaluating integrity of material), in accordance to the sensor configuration.

\section{ELECTROMAGNETIC SENSOR}

\subsection{Configuration and Operating Principle of Electronic Sensors}

The planar types of sensors, meander, mesh and interdigital, have been designed and fabricated Interdigital sensor the operating principle behind the interdigital sensor is very analogous to the one experiential in a parallel plate capacitor [11] exhibits the relationship between a parallel plate capacitor and an interdigital sensor, and how the evolution from the capacitor to a sensor is accomplished[12]. There is an electric field between the positive and negative electrodes as shown in figure 1 . These fields pass through the material under test (MUT). Therefore the material dielectric properties along with the electrode and material geometry affect the conductance and capacitance among the two electrodes.

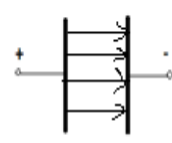

(a)

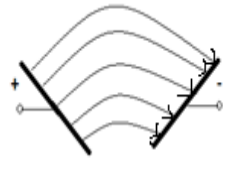

(b)

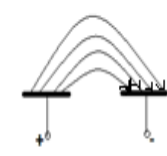

(c)
Fig.1. Transition from the capacitor to a sensor[11]

\subsection{Modelling and simulation of Interdigital Capacitor}

Seven electrodes are given the excitation as port 1 . The other six electrodes are connected to ground as port 2 . The electric field lines from the excitation electrodes originate and then terminate on the sensing electrodes. The electric field lines are affected by the distance of separation between the electrodes of same type that is termed to as wavelength. It is to be observed that the electric field lines vary for different wavelengths. Table 1 shows the dimensions of interdigital capacitor for two different wavelength. The change in frequency results in exponential decrease Impedance changes can be used to estimate and characterize the material properties as shown in figure 2 and 3 . These designs of interdigital capacitor show the capacitive characteristics that may be applied for measurement of dielectric properties of materials.

Table 1. Interdigital Capacitor specifications

\begin{tabular}{||l|l|l|}
\hline $\begin{array}{l}\text { Interdigital } \\
\text { Sensor }\end{array}$ & $\begin{array}{l}\text { Wavelength, } \\
\lambda(\mathrm{mm})\end{array}$ & $\begin{array}{l}\text { Length } \\
(\mathrm{mm})\end{array}$ \\
\hline 1 & 2 & 20 \\
\hline 2 & 3 & 40 \\
\hline
\end{tabular}




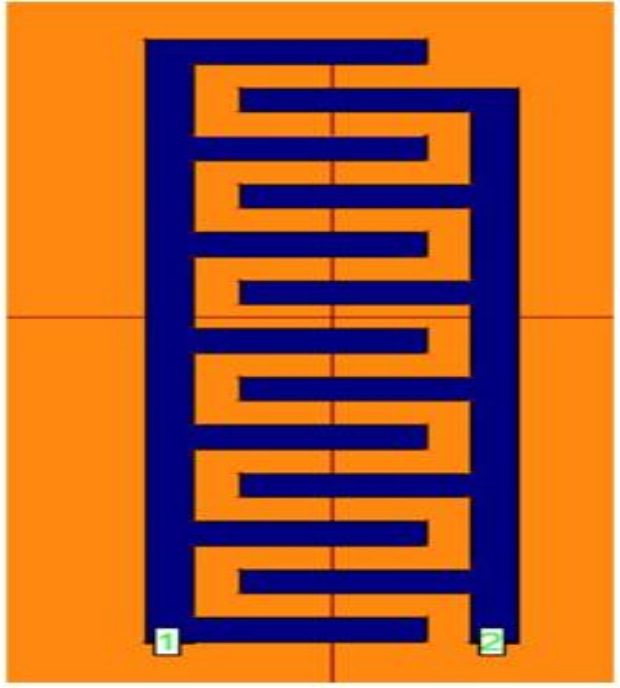

Fig 2.(a) Schematic of interdigital capacitor

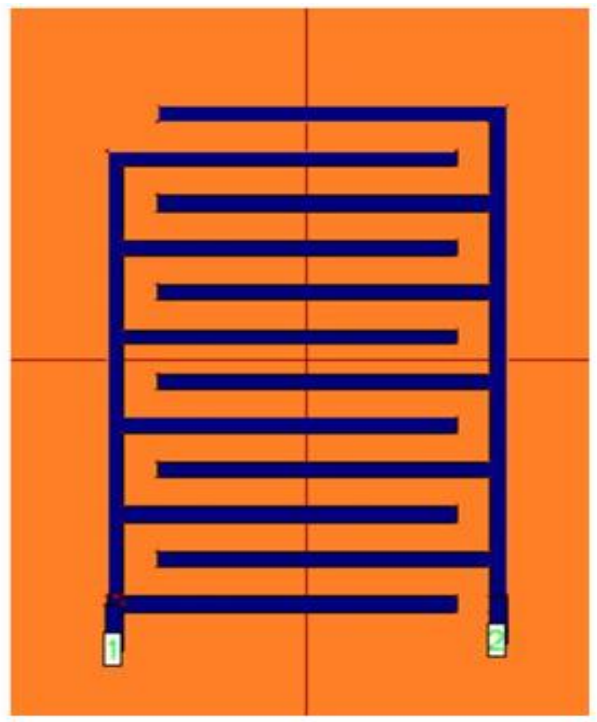

Fig 2.(b) Schematic of interdigital capacitor

\subsection{Modelling and simulation of hexagonal spiral inductor parameters}

Hexagonal spiral coil is patterned on FR4 with six, eight and ten turns. It is seen that the impedance of hexagonal spiral inductor increases with frequency, as shown in figure 4 and 5. Also, as the number of turns increases inductances increases yields in increase in the magnetic field. The variation in turns affects the impedance and inductance is as in table 2 . The electroplated copper traces are $1 \mathrm{~mm}$ thick. That is, the number of turns of the inductor is varied and the change in impedance with respect to frequency is observed. This results in generation of magnetic field because of the inductor will respond well to various materials. Since the thickness of the traces is much less than the skin depth, assumption that the current and voltage distribution is uniform through the thickness of the traces material.

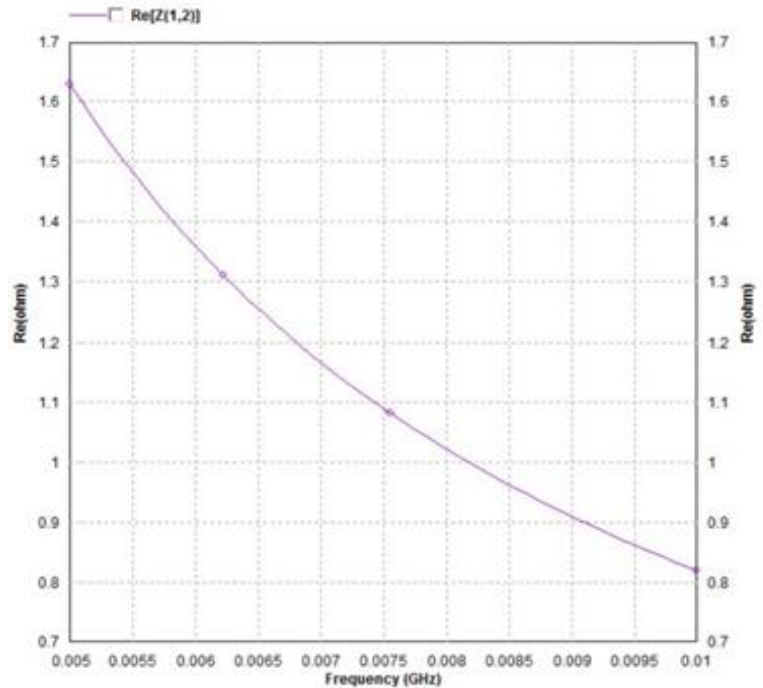

Fig.3. (a) Impedance variation with frequency

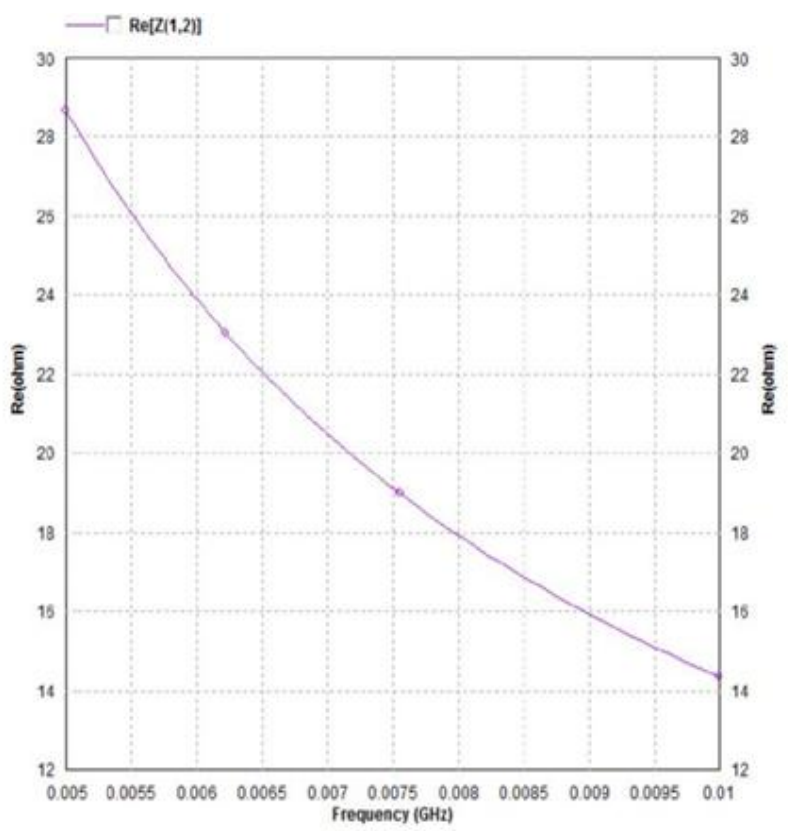

Fig.3. (b) Impedance variation with frequency

Table 2. Hexagonal spiral inductor specifications

\begin{tabular}{|c|c|c|}
\hline $\begin{array}{c}\text { Hexagonal } \\
\text { Sensor }\end{array}$ & No to turns & Inductance( $\boldsymbol{\mu H})$ \\
\hline 1 & 6 & 1.30 \\
\hline 2 & 8 & 2.16 \\
\hline 3 & 10 & 3.21 \\
\hline
\end{tabular}




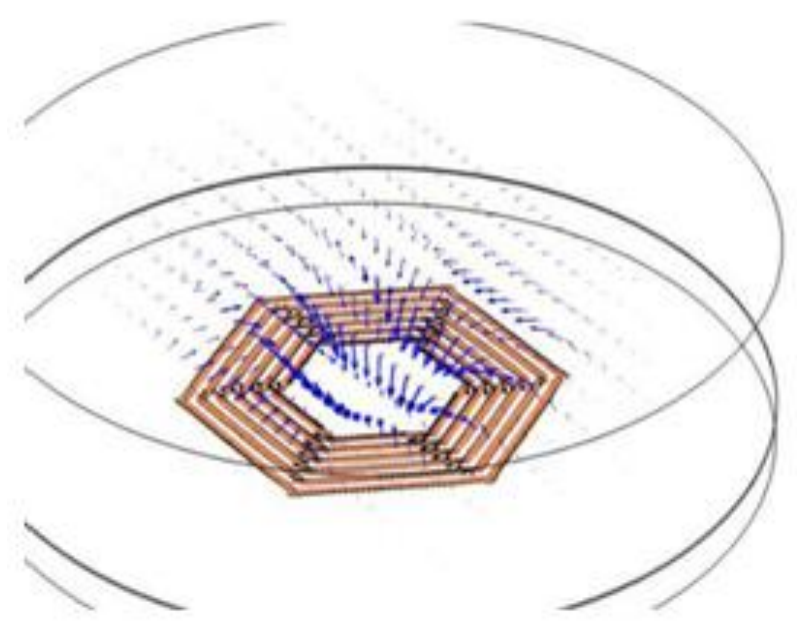

Fig.4. (a) Schematic of hexagonal spiral inductor with six turns

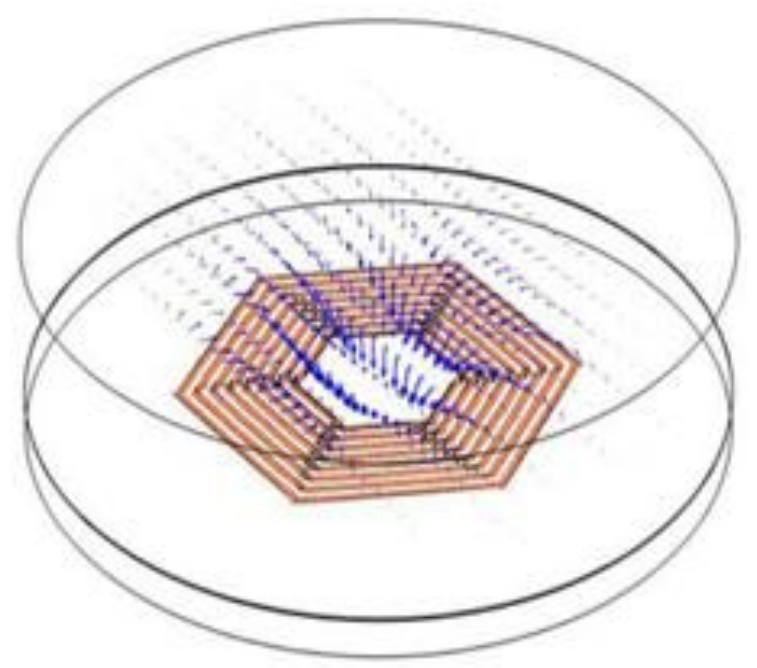

Fig 4 (b)Schematic of hexagonal spiral inductor with eight turns

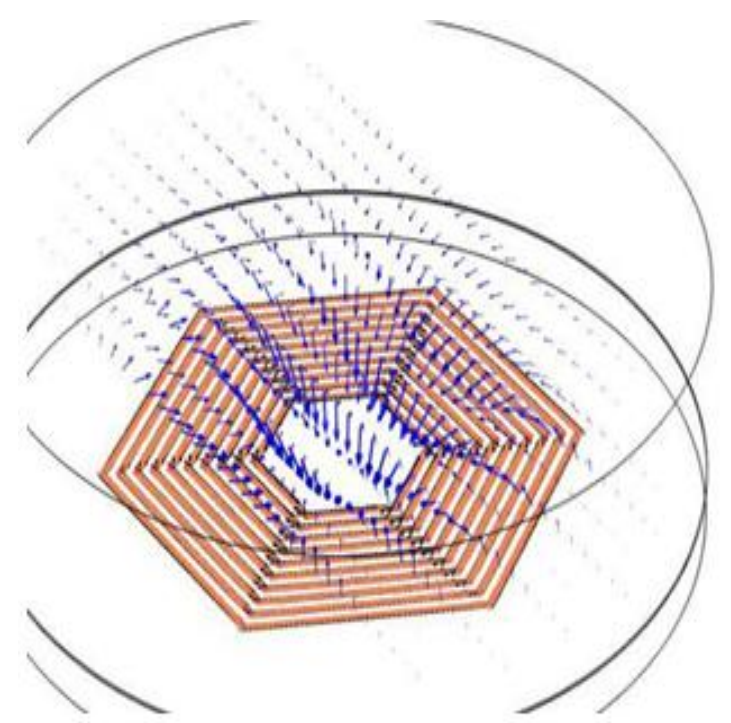

Fig.5. (a)Schematic of hexagonal spiral inductor with ten turns.

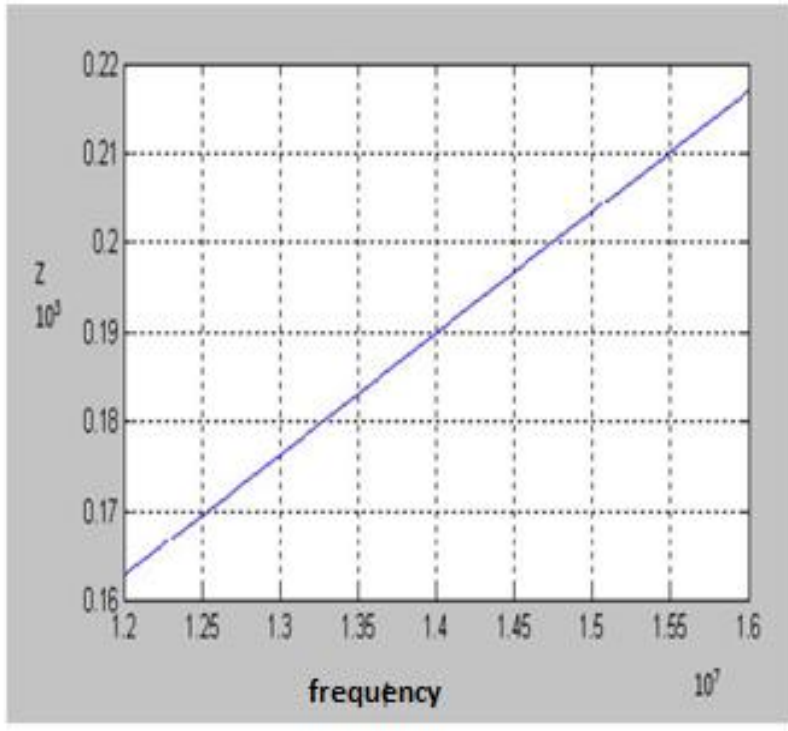

(b)Impedance variation with frequency

\section{CONCLUSION}

The observations show that the interdigital capacitor is able to generate electric field where as hexagonal spiral inductor produces a magnetic field this two may be used separately to determine various parameters. The variation in impedance will be sensed by the hexagonal spiral sensor due to variation in magnetic field. The magnetic field increases with increase in the number of turns for the inductor. Similarly the capacitive sensor produces electric field with variation of frequency effect will be utilized for parameter measurement. This paper introduces a novel hexagonal sensor that may be used for various parameter measurements.

\section{REFERENCES}

[1] Yamada S., Katou M., Iwahara M., Dawson F.P. Eddy current testing probe composed on planar coils. IEEE Transactions on Magnetics. 1995;31:3185-3187.

[2] Yamada S., Katou M., Iwahara M., Dawson F.P. Defect images by planar ECT probe of Meander-Mesh coils. IEEE Transactions on Magnetics. 1996; 32:49564958.

[3] Yamada S., Fujiki H., Iwahara M., Mukhopadhyay S.C., Dawson F.P. Investigation of printed wiring board testing by using planar coil type ECT probe. IEEE Transactions on Magnetics. 1997;33:3376-3378.

[4] Mukhopadhyay S.C., Yamada S., Iwahara M. Investigation of near-surface material properties using planar type meander coil. JSAEM Studies on Applied Electromagnetics and Mechanics. 2001;11:61-69.

[5] Mukhopadhyay S.C., Yamada S., Iwahara M. Evaluation of near- surface material properties using planar mesh type coils with post-processing from neural network model. International journal on Electromagnetic Nondestuctive Evaluation. 2002;23:181- 188.

[6] Mukhopadhyay S.C., Yamada S., Iwahara M. Inspection of electroplated materials - performance comparison with planar meander and mesh type magnetic sensor. International journal of Applied Electromagnetics and Mechanics. 2002;15:323-329. 
[7] Mukhopadhyay S.C. Quality inspection of electroplated materials using planar type micromagnetic sensors with post processing from neural network model. IEE Proceedings - Science, Measurement and Technology. 2002;149:165-171.

[8] Mukhopadhyay S.C., Woolley J.D.M., Sen Gupta G. Inspection of saxophone reeds employing a novel planar electromagntic sensing technique. Proceedings of 2011 International Instrumentation and Measurement Technology Conference. 2005:209-213. IEEE Catalog Number 05CH37627C, ISBN 0-7803-8880-1.

[9] Gooneratne C., Mukhopadhyay S.C., Purchas R., Sen Gupta G. Interaction of planar electromagnetic sensors with pork belly cuts. Proceedings of $1^{\text {st }}$ International Conference on Sensing Technology. 2005:519-526.

[10] Mamishev A., Sundara-Rajan K., Yang F., Du Y., Zahn M. Interdigital sensors and transducers. Proceedings of the IEEE. 2004;92:808-845.

[11] Chretiennot, T. ; LAAS, Toulouse, France ; Dubuc, D. ; Grenier, K." Optimized electromagnetic interaction microwave resonator/microfluidic channel for enhanced liquid bio-sensor "6-10 Oct. 2013 Page(s):464 - 467.

[12] Mamishev A., Sundara-Rajan K., Yang F., Du Y., Zahn M. Interdigital sensors and transducers. Proceedings of the IEEE. 2004; 92:808-845. 